\title{
Rotation number and lifts of a Fuchsian action of the modular group on the circle
}

\author{
Yoshifumi MATSUDA*†
}

\begin{abstract}
We characterize the semi-conjugacy class of a Fuchsian action of the modular group on the circle in terms of rotation numbers of two standard generators and that of their product. We also show that among lifts of a Fuchsian action of the modular group, only 5-fold lift admits a similar characterization. These results indicate similarity and difference between rotation number and linear character.
\end{abstract}

Keywords: Rotation number, modular group, group actions on the circle

2010 MSC: Primary 37E45; Secondary 37C85, 37E10

\section{Introduction}

Rotation number of an orientation-preserving homeomorphism of the circle has similar properties to absolute value of the trace of an element in $\operatorname{PSL}(2, \mathbb{R})$. For example, they are invariant under conjugation and furthermore, Jørgensen's criterion of discreteness for subgroups of $\operatorname{PSL}(2, \mathbb{R})[11$, Theorem 2], which can be described in terms of absolute value of the trace, has an analogue for the group of real analytic diffeomorphisms of the circle (see [13, Theorem 1.2]). In this article, we give another similarity between rotation number and linear character from a viewpoint given by D. Calegari and A. Walker [5].

\subsection{Rotation number}

We denote by Homeo+ $\left(\mathbb{S}^{1}\right)$ the group of orientation-preserving homeomorphisms of the circle. We regard the circle $\mathbb{S}^{1}$ as the quotient $\mathbb{R} / \mathbb{Z}$ and denote by $p: \mathbb{R} \rightarrow$ $\mathbb{S}^{1}$ the projection. Let $\widetilde{\mathrm{HomeO}_{+}}\left(\mathbb{S}^{1}\right)$ be the group of lifts of orientation-preserving homeomorphisms to $\mathbb{R}$, namely, homeomorphisms of $\mathbb{R}$ commuting with integral translations.

${ }^{*}$ Department of Physics and Mathematics, College of Science and Engineering, Aoyama Gakuin University 5-10-1 Fuchinobe, Chuo-ku, Sagamihara-shi, Kanagawa 252-5258 Japan, ymatsuda@gem.aoyama.ac.jp

${ }^{\dagger}$ Partly supported by JSPS KAKENHI Grant Number 25800036. 
For $\tilde{f} \in \widetilde{\mathrm{HomeO}_{+}}\left(\mathbb{S}^{1}\right)$, we define the translation number $\widetilde{\operatorname{rot}}(\tilde{f}) \in \mathbb{R}$ of $\tilde{f}$ by

$$
\widetilde{\operatorname{rot}}(\tilde{f})=\lim _{n \rightarrow \infty} \frac{(\tilde{f})^{n}(\tilde{x})-\tilde{x}}{n},
$$

where $\tilde{x} \in \mathbb{R}$. Note that the limit exists and does not depend on the choice of a point $\tilde{x} \in \mathbb{R}$. For $f \in \mathrm{Homeo}_{+}\left(\mathbb{S}^{1}\right)$, we define the rotation number $\operatorname{rot}(f) \in \mathbb{R} / \mathbb{Z}$ of $f$ by

$$
\operatorname{rot}(f)=\widetilde{\operatorname{rot}}(\tilde{f}) \bmod \mathbb{Z}
$$

where $\tilde{f} \in \widetilde{\text { Homeo }_{+}}\left(\mathbb{S}^{1}\right)$ is a lift of $f$ to $\mathbb{R}$.

Among several properties of rotation number, we recall that $\operatorname{rot}(f)=\frac{p}{q}$, where $\frac{p}{q}$ is a reduced fraction if and only if $f$ has a period point of period $q$. In particular, $\operatorname{rot}(f)=0$ if and only if $f$ has a fixed point (see for example [9] in detail and other properties of rotation number).

\subsection{Lifts of a group action on the circle}

For a group $\Gamma$, we denote by $\mathrm{R}(\Gamma)$ the space of homomorphisms from $\Gamma$ to Homeo $_{+}\left(\mathbb{S}^{1}\right)$. We equip $\mathrm{R}(\Gamma)$ with the uniform convergence topology on generators if necessary.

We define a lift of a group action on the circle.

Let $k \geq 2$ be a positive integer and denote by $p_{k}: \mathbb{S}^{1} \rightarrow \mathbb{S}^{1}$ the $k$-fold covering map. For a group $\Gamma$, a homomorphism $\phi \in \mathrm{R}(\Gamma)$ is a $k$-fold lift of a homomorphism $\psi \in \mathrm{R}(\Gamma)$ if $p_{k} \circ \phi(\gamma)=\psi(\gamma) \circ p_{k}$ for every $\gamma \in \Gamma$.

We remark that if $\phi \in \mathrm{R}(\Gamma)$ is a $k$-fold lift of a homomorphism $\psi \in \mathrm{R}(\Gamma)$, then we have $k \operatorname{rot}(\phi(\gamma))=\operatorname{rot}(\psi(\gamma))$ for every $\gamma \in \Gamma$.

\subsection{Semi-conjugacy class}

Semi-conjugacy between two actions of a group on the circle has been defined in several ways (see [8], [9], 1]). In this paper, we follow the way presented in 3.

For $\phi_{1}, \phi_{2} \in \mathrm{R}(\Gamma)$, we say that $\phi_{1}$ is semi-conjugate to $\phi_{2}$ if there exists a continuous degree-one monotone map such that $h \circ \phi_{1}(\gamma)=\phi_{2}(\gamma) \circ h$ for every $\gamma \in \Gamma$. Here, a map $h: \mathbb{S}^{1} \rightarrow \mathbb{S}^{1}$ is called a degree-one monotone map if it admits a lift $\tilde{h}: \mathbb{R} \rightarrow \mathbb{R}$ commuting with integral translations, and nondecreasing on $\mathbb{R}$.

Note that semi-conjugacy is not symmetric and is not an equivalence relation. We consider the equivalence relation generated by semi-conjugacy, which is called monotone equivalence in [3]. We call the monotone equivalence class of $\phi \in \mathrm{R}(\Gamma)$ the semi-conjugacy class of $\phi$. Note that if two minimal homomorphisms belong to the same semi-conjugacy class, then they are topologically conjugate. We define the semi-conjugacy class of an orientation-preserving homeomorphism of the circle in a similar way. 
A classical result due to $\mathrm{H}$. Poincaré says that two homeomorphisms are in the same semi-conjugacy class if and only if their rotation numbers coincide, which is similar to the fact that two matrices in $\mathrm{SL}(2, \mathbb{R}) \backslash\{ \pm E\}$ are conjugate if and only if their traces coincide.

As for group actions, however, $\phi_{1}, \phi_{2} \in \mathrm{R}(\Gamma)$ do not belong to the same semi-conjugacy class if we only suppose that $\operatorname{rot}\left(\phi_{1}(\gamma)\right)=\operatorname{rot}\left(\phi_{2}(\gamma)\right)$ for every $\gamma$. It can be seen by considering Fuchsian actions corresponding to hyperbolic structures on 2-orbifolds (see for example [6] about 2-orbifolds and hyperbolic structures on them).

\subsection{Fuchsian actions}

Let $\mathcal{O}$ be a compact, connected, oriented 2-orbifold with negative orbifold Euler characteristic $\chi^{\text {orb }}(\mathcal{O})<0$. For each hyperbolic structure on the interior of $\mathcal{O}$ compatible with the orientation of $\mathcal{O}$, we have a homomorphism from the orbifold fundamental group $\pi_{1}^{\text {orb }}(\mathcal{O})$ to $\operatorname{PSL}(2, \mathbb{R})$ by identifying the universal cover $\tilde{\mathcal{O}}$ with the hyperbolic plane $\mathbb{H}^{2}$. By considering the action on the ideal boundary $\partial \mathbb{H}^{2} \simeq \mathbb{S}^{1}$, we obtain a homomorphism $\phi_{\mathcal{O}} \in \mathrm{R}\left(\pi_{1}^{\text {orb }}(\mathcal{O})\right)$. We call such a homomorphism a Fuchsian action associated to $\mathcal{O}$. Note that the semi-conjugacy class of a Fuchsian action associated to a fixed 2-orbifold $\mathcal{O}$ is independent of the choice of a hyperbolic structure and that a Fuchsian action corresponding to a hyperbolic structure with finite area is minimal.

In general, we cannot characterize the semi-conjugacy class of a Fuchsian action only by rotation numbers of all elements. In fact, for a Fuchsian action $\phi_{S}$ associated to a compact, connected, oriented surface $S$ with negative Euler characteristic, the homeomorphism $\phi_{S}(\gamma)$ has a fixed point for every $\gamma \in \Gamma$ but there is no global fixed point. This means that $\operatorname{rot}\left(\phi_{S}(\gamma)\right)=0$ for every $\gamma \in \Gamma$ but the Fuchsian action $\phi_{S}$ does not belong to the semi-conjugacy class of the trivial action.

Now we show, however, that we can characterize the semi-conjugacy classes of a Fuchsian action of a specific 2-orbifold and its certain lift by only rotation numbers of finite elements.

\subsection{Main result}

We focus on a special 2-orbifold. Let $\mathcal{O}_{2,3}$ be the 2-orbifold which is obtained from a 2 -disk by making two cone-points of orders 2,3 . Note that the interior of $\mathcal{O}_{2,3}$ is homeomorphic to $\mathbb{H}^{2} / \operatorname{PSL}(2 ; \mathbb{Z})$ and $\pi_{1}^{\text {orb }}\left(\mathcal{O}_{2,3}\right)$ is isomorphic to the modular group $\operatorname{PSL}(2, \mathbb{Z})$. We fix a presentation

$$
\pi_{1}^{\text {orb }}\left(\mathcal{O}_{2,3}\right)=\left\langle\alpha, \beta \mid \alpha^{2}=\beta^{3}=1\right\rangle \cong \mathbb{Z}_{2} * \mathbb{Z}_{3}, .
$$

where $\alpha=\left[\begin{array}{cc}0 & -1 \\ 1 & 0\end{array}\right]$ and $\beta=\left[\begin{array}{cc}1 & 1 \\ -1 & 0\end{array}\right]$. Let $\phi_{\mathcal{O}_{2,3}}$ be a Fuchsian action of $\mathcal{O}_{2,3}$ which is equal to the action by linear fractional transformations on 
$\mathbb{R} \cup\{\infty\} \simeq \mathbb{S}^{1}$. It follows that

$$
\begin{aligned}
& \phi_{\mathcal{O}_{2,3}}(\alpha)(0)=\infty, \quad \phi_{\mathcal{O}_{2,3}}(\alpha)(\infty)=0 \\
& \phi_{\mathcal{O}_{2,3}}(\beta)(0)=\infty, \quad \phi_{\mathcal{O}_{2,3}}(\beta)(\infty)=-1, \quad \phi_{\mathcal{O}_{2,3}}(\beta)(-1)=0 \text { and } \\
& \phi_{\mathcal{O}_{2,3}}(\alpha \beta)(0)=0 .
\end{aligned}
$$

Hence we have

$$
\left(\operatorname{rot}\left(\phi_{\mathcal{O}_{2,3}}(\alpha)\right), \operatorname{rot}\left(\phi_{\mathcal{O}_{2,3}}(\beta)\right), \operatorname{rot}\left(\phi_{\mathcal{O}_{2,3}}(\alpha \beta)\right)\right)=\left(\frac{1}{2}, \frac{1}{3}, 0\right) .
$$

It follows from the presentation of $\pi_{1}^{\text {orb }}\left(\mathcal{O}_{2,3}\right)$ that there exists a $k$-fold lift $\phi_{\mathcal{O}_{2,3}}^{(k)}$ of $\phi_{\mathcal{O}_{2,3}}$ if and only if $k \equiv \pm 1 \bmod 6$ and that such a lift is unique if it exists. We also have

$$
\begin{aligned}
& \left(\operatorname{rot}\left(\phi_{\mathcal{O}_{2,3}}^{(k)}(\alpha)\right), \operatorname{rot}\left(\phi_{\mathcal{O}_{2,3}}^{(k)}(\beta)\right), \operatorname{rot}\left(\phi_{\mathcal{O}_{2,3}}^{(k)}(\alpha \beta)\right)\right) \\
& = \begin{cases}\left(\frac{1}{2}, \frac{1}{3}, \frac{k-1}{k}\right) & (k \equiv 1 \bmod 6) \\
\left(\frac{1}{2}, \frac{2}{3}, \frac{1}{k}\right) & (k \equiv-1 \bmod 6)\end{cases}
\end{aligned}
$$

Now we are ready to state the main result.

Theorem 1.1. Let $\phi \in \mathrm{R}\left(\pi_{1}^{\text {orb }}\left(\mathcal{O}_{2,3}\right)\right)$.

1. If $\left(\operatorname{rot}(\phi(\alpha)), \operatorname{rot}(\phi(\beta)), \operatorname{rot}(\phi(\alpha \beta))=\left(\frac{1}{2}, \frac{1}{3}, 0\right)\right.$, then $\phi$ belongs to the semi-conjugacy class of a Fuchsian action $\phi_{\mathcal{O}_{2,3}}$.

2. If $\left(\operatorname{rot}(\phi(\alpha)), \operatorname{rot}(\phi(\beta)), \operatorname{rot}(\phi(\alpha \beta))=\left(\frac{1}{2}, \frac{2}{3}, \frac{1}{5}\right)\right.$, then $\phi$ belongs to the semi-conjugacy class of the 5 -fold lift $\phi_{\mathcal{O}_{2,3}}^{(5)}$ of a Fuchsian action $\phi_{\mathcal{O}_{2,3}}$.

Remark 1.2. 1. Theorem 1.1 cannot be generalized to the other lifts of $\phi_{\mathcal{O}_{2,3}}$. Indeed for each positive integer $k \geq 2$ we denote by $\mathcal{O}_{2,3, k}$ a compact, connected, oriented 2-orbifold which is obtained from a 2 -sphere by making three cone-points of orders $2,3, k$. Now suppose that $k \equiv \pm 1$ $\bmod 6$ and $k \neq 5$. Then we have $\chi^{\text {orb }}\left(\mathcal{O}_{2,3, k}\right)<0$. Let $\phi_{\mathcal{O}_{2,3, k}} \in$ $\mathrm{R}\left(\pi_{1}^{\text {orb }}\left(\mathcal{O}_{2,3, k}\right)\right)$ be a Fuchsian action of $\mathcal{O}_{2,3, k}$. For a suitable presentation

$$
\pi_{1}^{\text {orb }}\left(\mathcal{O}_{2,3, k}\right)=\left\langle\alpha, \beta, \gamma \mid \alpha^{2}=\beta^{3}=\gamma^{k}=\alpha \beta \gamma=1\right\rangle,
$$

we have

$$
\begin{aligned}
& \left(\operatorname{rot}\left(\phi_{\mathcal{O}_{2,3, k}}(\alpha)\right), \operatorname{rot}\left(\phi_{\mathcal{O}_{2,3, k}}(\beta)\right), \operatorname{rot}\left(\phi_{\mathcal{O}_{2,3, k}}(\gamma)\right)\right) \\
= & \left(\frac{1}{2}, \frac{1}{3}, \frac{1}{k}\right)
\end{aligned}
$$


and hence

$$
\begin{aligned}
& \left(\operatorname{rot}\left(\phi_{\mathcal{O}_{2,3, k}}(\alpha)\right), \operatorname{rot}\left(\phi_{\mathcal{O}_{2,3, k}}(\beta)\right), \operatorname{rot}\left(\phi_{\mathcal{O}_{2,3, k}}(\alpha \beta)\right)\right) \\
= & \left(\frac{1}{2}, \frac{1}{3}, \frac{k-1}{k}\right)
\end{aligned}
$$

Let $q$ be the homomorphism from $\pi_{1}^{\text {orb }}\left(\mathcal{O}_{2,3}\right)$ onto $\pi_{1}^{\text {orb }}\left(\mathcal{O}_{2,3, k}\right)$ such that $q(\alpha)=\alpha$ and $q(\beta)=\beta$ and let $\iota$ be the automorphism of $\pi_{1}^{\text {orb }}\left(\mathcal{O}_{2,3}\right)$ such that $\iota(\alpha)=\alpha$ and $\iota(\beta)=\beta^{-1}$. We define a homomorphism $\hat{\phi}_{\mathcal{O}_{2,3, k}} \in$ $\mathrm{R}\left(\pi_{1}^{\text {orb }}\left(\mathcal{O}_{2,3}\right)\right)$ by

$$
\hat{\phi}_{\mathcal{O}_{2,3, k}}= \begin{cases}\phi_{\mathcal{O}_{2,3, k}} \circ q & (k \equiv 1 \bmod 6) \\ \phi_{\mathcal{O}_{2,3, k}} \circ q \circ \iota & (k \equiv-1 \bmod 6),\end{cases}
$$

Since both $\phi_{\mathcal{O}_{2,3, k}}$ and $\phi_{\mathcal{O}_{2,3}}$ are minimal, it follows that both $\hat{\phi}_{\mathcal{O}_{2,3, k}}$ are $\phi_{\mathcal{O}_{2,3}}^{(k)}$ are also minimal. It follows that

$$
\begin{aligned}
& \left(\operatorname{rot}\left(\hat{\phi}_{\mathcal{O}_{2,3, k}}(\alpha)\right), \operatorname{rot}\left(\hat{\phi}_{\mathcal{O}_{2,3, k}}(\beta)\right), \operatorname{rot}\left(\hat{\phi}_{\mathcal{O}_{2,3, k}}(\alpha \beta)\right)\right) \\
= & \left(\operatorname{rot}\left(\phi_{\mathcal{O}_{2,3}}^{(k)}(\alpha)\right), \operatorname{rot}\left(\phi_{\mathcal{O}_{2,3}}^{(k)}(\beta)\right), \operatorname{rot}\left(\phi_{\mathcal{O}_{2,3}}^{(k)}(\alpha \beta)\right)\right) .
\end{aligned}
$$

Note that if $k \equiv-1 \bmod 6$, then we have

$$
\begin{aligned}
& \operatorname{rot}\left(\hat{\phi}_{\mathcal{O}_{2,3, k}}(\alpha \beta)\right) \\
= & \operatorname{rot}\left(\phi_{\mathcal{O}_{2,3, k}}\left(\alpha \beta^{-1}\right)\right) \\
= & \operatorname{rot}\left(\phi_{\mathcal{O}_{2,3, k}}(\beta)\left(\phi_{\mathcal{O}_{2,3, k}}(\alpha \beta)\right)^{-1}\left(\phi_{\mathcal{O}_{2,3, k}}(\beta)\right)^{-1}\right) \\
= & -\operatorname{rot}\left(\phi_{\mathcal{O}_{2,3, k}}(\alpha \beta)\right) .
\end{aligned}
$$

On the other hand $\hat{\phi}_{\mathcal{O}_{2,3, k}}$ and $\phi_{\mathcal{O}_{2,3}}^{(k)}$ do not belong to the same semiconjugacy class. Indeed if they belonged the same conjugacy class, then they would be topologically conjugate by minimality. However this contradicts the fact that

$$
\hat{\phi}_{\mathcal{O}_{2,3, k}}\left((\alpha \beta)^{k}\right)=\mathrm{id} \neq \phi_{\mathcal{O}_{2,3}}^{(k)}\left((\alpha \beta)^{k}\right) .
$$

2. We can prove Theorem 1.1 (1) by generalizing the notion of the bounded Euler number defined in 2] to actions of 2-orbifold groups. It will be indicated in a forthcoming paper together with generalizations of Theorem 1.1 to actions of other 2-orbifold groups.

3. Theorem 1.1 can be considered as a weak analogue of the following classical theorem about linear character [7, which we write in a specified form. Let $F\langle\alpha, \beta\rangle$ be a free group of rank two with a basis $\alpha, \beta$.

Theorem 1.3. Let $\phi, \psi: F\langle\alpha, \beta\rangle \rightarrow \mathrm{SL}(2, \mathbb{R})$ be homomorphisms. If we have

$$
\begin{aligned}
& (\operatorname{tr}(\phi(\alpha)), \operatorname{tr}(\phi(\beta)), \operatorname{tr}(\phi(\alpha \beta))) \\
= & (\operatorname{tr}(\psi(\alpha)), \operatorname{tr}(\psi(\beta)), \operatorname{tr}(\psi(\alpha \beta))) \\
= & (x, y, z)
\end{aligned}
$$


with $x^{2}+y^{2}+z^{2}-x y z \neq 4$, then $\phi$ and $\psi$ are conjugate by an element of $\operatorname{PSL}(2, \mathbb{R})$.

4. When the author mentioned Theorem 1.1 in his talk given in the conference "Geometry and Foliations 2013", E. Ghys informed us the following theorem about linear character.

Theorem 1.4. [10, Example 8.2] Let $F_{m}$ be a free group of rank $m \geq 2$. For every positive integer $n$, there exist mutually non-conjugate elements $w_{1}, \ldots, w_{n}$ of $F_{m}$ such that for every homomorphism $\phi: F_{m} \rightarrow \mathrm{SL}(2, \mathbb{R})$, we have

$$
\operatorname{tr}\left(\phi\left(w_{1}\right)\right)=\cdots=\operatorname{tr}\left(\phi\left(w_{n}\right)\right)
$$

After that, he asked the following question.

Question 1.5. Does the following analogue of Theorem 1.4 hold for Homeo $_{+}\left(\mathbb{S}^{1}\right)$ ? Namely, for every positive integer $m \geq 2$ and every positive integer $n$, does there exist mutually non-conjugate elements $w_{1}, \ldots, w_{n}$ of $F_{m}$ such that for every homomorphism $\phi \in \mathrm{R}\left(F_{m}\right)$, we have

$$
\operatorname{rot}\left(\phi\left(w_{1}\right)\right)=\cdots=\operatorname{rot}\left(\phi\left(w_{n}\right)\right) ?
$$

Note that D. Calegari asked this question for the case where $m=2, n=2$ and $w_{2}$ is fixed as the identity element [4].

\section{Proof of Theorem 1.1}

For $r_{1}, r_{2}, r_{3} \in \mathbb{R} / \mathbb{Z}$, we put

$$
\begin{aligned}
& \mathrm{R}\left(r_{1}, r_{2}, r_{3}\right) \\
= & \left\{\phi \in \mathrm{R}\left(\pi_{1}^{\text {orb }}\left(\mathcal{O}_{2,3}\right)\right) \mid\left(\operatorname{rot}(\phi(\alpha)), \operatorname{rot}(\phi(\beta)), \operatorname{rot}(\phi(\alpha \beta))=\left(r_{1}, r_{2}, r_{3}\right)\right\} .\right.
\end{aligned}
$$

\subsection{Proof of (1)}

Let $\phi \in \mathrm{R}\left(\frac{1}{2}, \frac{1}{3}, 0\right)$. The following sufficient condition for belonging to the same semi-conjugacy class given in [12] is a corollary of a criterion in [14].

Proposition 2.1. [12, Corollary 7.5] Let $\Gamma$ be a group and $U \subset \mathrm{R}(\Gamma)$ be connected. Suppose that $\operatorname{rot}\left(\phi_{1}(\gamma)\right)=\operatorname{rot}\left(\phi_{2}(\gamma)\right)$ for every $\phi_{1}, \phi_{2} \in U$ and every $\gamma \in \Gamma$, then $U$ is contained in a single semi-conjugacy class.

In view of Proposition 2.1, it suffices to show the following.

Lemma 2.2. $\operatorname{rot}(\phi(\gamma))=\operatorname{rot}\left(\phi_{\mathcal{O}_{2,3}}(\gamma)\right)$ for every $\gamma \in \pi_{1}^{\text {orb }}\left(\mathcal{O}_{2,3}\right)$. 
Lemma 2.3. The space $\mathrm{R}\left(\frac{1}{2}, \frac{1}{3}, 0\right)$ is path-connected.

Proof of Lemma 2.2, We denote by $\tilde{a}$ (resp. $\tilde{b})$ the lift of $\phi(\alpha)($ resp. $\phi(\beta))$ with $\widetilde{\operatorname{rot}}(\tilde{a})=\frac{1}{2}\left(\operatorname{resp} . \widetilde{\operatorname{rot}}(\tilde{b})=\frac{1}{3}\right)$. Since $0<\widetilde{\operatorname{rot}}(\tilde{a})<1$, we have

$$
\tilde{x}<\tilde{a}(\tilde{x})<\tilde{x}+1
$$

for every $\tilde{x} \in \mathbb{R}$. Hence we have

$$
\tilde{b}(\tilde{x})<(\tilde{a} \tilde{b})(\tilde{x})<\tilde{b}(\tilde{x})+1
$$

for every $\tilde{x} \in \mathbb{R}$. This implies that

$$
\frac{1}{3}=\widetilde{\operatorname{rot}}(\tilde{b}) \leq \widetilde{\operatorname{rot}}(\tilde{a} \tilde{b}) \leq \widetilde{\operatorname{rot}}(\tilde{b})+1=\frac{4}{3} .
$$

Since $\operatorname{rot}(\phi(\alpha \beta))=0$, we have $\widetilde{\operatorname{rot}}(\tilde{a} \tilde{b})=1$. Then there exists a point $\tilde{x_{0}} \in \mathbb{R}$ such that $(\tilde{a} \tilde{b})\left(\tilde{x}_{0}\right)=\tilde{x}_{0}+1$. Since both $\tilde{a}^{2}$ and $\tilde{b}^{3}$ are the translation by one, we have

$$
\tilde{x}_{0}<\tilde{a}\left(\tilde{x}_{0}\right)=\tilde{b}\left(\tilde{x}_{0}\right)<\tilde{b}^{2}\left(\tilde{x}_{0}\right)<\tilde{x}_{0}+1
$$

We put

$$
\begin{aligned}
I & =p\left(\left[\tilde{x}_{0}, \tilde{b}\left(\tilde{x}_{0}\right)\right]\right. \text { and } \\
J & =p\left(\left[\tilde{b}\left(\tilde{x}_{0}\right), \tilde{x}_{0}+1\right]\right) .
\end{aligned}
$$

Then we have

$$
\begin{aligned}
\phi(\alpha)(J) & =I \text { and } \\
\phi\left(\beta^{ \pm 1}\right)(I) & \subset J .
\end{aligned}
$$

We claim that if $\gamma \in \Gamma$ is not conjugate to a power of $\alpha, \beta$, then there exists a closed interval $K \subset \mathbb{S}^{1}$ such that $\phi(\gamma)(K) \subset K$. Indeed by taking conjugates if necessary, we may assume that $\gamma=\alpha \beta^{e_{1}} \cdots \alpha \beta^{e_{n}}$, where $e_{i} \in \pm 1$ for $i \in\{1, \ldots, n\}$. Then we have $\phi(\gamma)(I) \subset I$.

This implies that if $\gamma$ is not conjugate to a power of $\alpha, \beta$, then $\operatorname{rot}(\phi(\gamma))=0$. This finishes the proof of the lemma.

Proof of Lemma 2.3. Let $\phi_{0}, \phi_{1} \in \mathrm{R}\left(\frac{1}{2}, \frac{1}{3}, 0\right)$. We show that there exists a path in $\mathrm{R}\left(\frac{1}{2}, \frac{1}{3}, 0\right)$ from $\phi_{0}$ to $\phi_{1}$. For $t \in\{0,1\}$, we denote by $\tilde{a}_{t}$ (resp. $\tilde{b}_{t}$ ) the lift of $\phi_{t}(\alpha)\left(\operatorname{resp} . \phi_{t}(\beta)\right)$ with $\widetilde{\operatorname{rot}}\left(\tilde{a}_{t}\right)=\frac{1}{2}\left(\operatorname{resp} . \widetilde{\operatorname{rot}}\left(\tilde{b}_{t}\right)=\frac{1}{3}\right)$. By taking conjugates, we may assume that both $\phi_{0}(b)$ and $\phi_{1}(b)$ are the rotation by $\frac{1}{3}$, 
and that $\left(\tilde{a}_{t} \tilde{b}_{t}\right)(0)=1$ for $t \in\{0,1\}$. We take a path $\left\{\tilde{a}_{t}\right\}_{t \in[0,1]}$ in $\widetilde{\text { Homeo }}+\left(\mathbb{S}^{1}\right)$ from $\tilde{a}_{0}$ to $\tilde{a}_{1}$ such that $\left(\tilde{a}_{t}\right)\left(\frac{1}{3}\right)=1$ and $\left(\tilde{a}_{t}\right)^{2}$ is the translation by one. We denote by $a_{t} \in \mathrm{Homeo}_{+}\left(\mathbb{S}^{1}\right)$ the projection of $\tilde{a}_{t}$. Then the path $\left\{\phi_{t}\right\}_{t \in[0,1]}$ in $\mathrm{R}\left(\frac{1}{2}, \frac{1}{3}, 0\right)$ defined by the condition that $\phi_{t}(\alpha)=a_{t}$ and $\phi_{t}(\beta)$ is the rotation by $\frac{1}{3}$ is a desired one.

\subsection{Proof of (2)}

Let $\phi \in \mathrm{R}\left(\frac{1}{2}, \frac{2}{3}, \frac{1}{5}\right)$. Then $\phi$ has no finite orbits. In fact if there were finite orbits, then the map rot $\circ \phi: \mathbb{Z}_{2} * \mathbb{Z}_{3} \rightarrow \mathbb{R} / \mathbb{Z}$ must be a homomorphism, which is impossible since $\operatorname{rot}(\phi(\alpha))=\frac{1}{2}, \operatorname{rot}(\phi(\beta))=\frac{2}{3}$ and $\operatorname{rot}(\phi(\alpha \beta))=\frac{1}{5}$. Therefore the action $\phi$ admits a unique minimal set, either a Cantor set or the whole circle. Passing to a semi-conjugate action, we may assume the latter, that is, the action is minimal.

By Theorem 1.1(1), it suffices to show that $\phi$ is the 5-fold lift of some action, namely, there exists a homeomorphism $\theta \in \mathrm{Homeo}_{+}\left(\mathbb{S}^{1}\right)$ which is $\phi\left(\pi_{1}^{\text {orb }}\left(\mathcal{O}_{2,3}\right)\right)$ equivariant and periodic of period 5 .

We denote by $\tilde{a}(\operatorname{resp} . \tilde{b})$ the lift of $\phi(\alpha)(\operatorname{resp} . \phi(\beta))$ with $\widetilde{\operatorname{rot}}(\tilde{a})=\frac{1}{2}$ $\left(\right.$ resp. $\left.\widetilde{\operatorname{rot}}(\tilde{b})=\frac{2}{3}\right)$. Since $0<\widetilde{\operatorname{rot}}(\tilde{a})<1$, we have

$$
\tilde{x}<\tilde{a}(\tilde{x})<\tilde{x}+1
$$

for every $\tilde{x} \in \mathbb{R}$. Hence we have

$$
\tilde{b}(\tilde{x})<(\tilde{a} \tilde{b})(\tilde{x})<\tilde{b}(\tilde{x})+1
$$

for every $\tilde{x} \in \mathbb{R}$. This implies that

$$
\frac{2}{3}=\widetilde{\operatorname{rot}}(\tilde{b}) \leq \widetilde{\operatorname{rot}}(\tilde{a} \tilde{b}) \leq \widetilde{\operatorname{rot}}(\tilde{b})+1=\frac{5}{3} .
$$

Since $\operatorname{rot}(\phi(\alpha \beta))=\frac{1}{5}$, we have $\widetilde{\operatorname{rot}}(\tilde{a} \tilde{b})=\frac{6}{5}$. We denote by $\widetilde{a b}$ the lift of $\phi(\alpha \beta)$ with $\widetilde{\operatorname{rot}}(\tilde{a b})=\frac{1}{5}$. Then there exists a point $\tilde{x_{0}} \in \mathbb{R}$ such that $(\tilde{a b})^{5}\left(\tilde{x}_{0}\right)=\tilde{x}_{0}+1$. Note that $\tilde{a} \tilde{b}(\tilde{x})=\tilde{a b}(\tilde{x})+1$ for every $\tilde{x} \in \mathbb{R}$.

Lemma 2.4. We have the following.

(1) $\tilde{a}(\tilde{x})<\tilde{b}(\tilde{x})$ for every $\tilde{x} \in \mathbb{R}$.

(2) $(\tilde{a b})^{2} \tilde{a}(\tilde{x})<\tilde{x}+1$ for every $\tilde{x} \in \mathbb{R}$.

(3) $(\tilde{a b})^{l}\left(\tilde{x}_{0}\right)<\tilde{b}(\tilde{a b})^{l+2}\left(\tilde{x}_{0}\right)-1<\tilde{b}^{2}(\tilde{a b})^{l+4}\left(\tilde{x}_{0}\right)-2<(\tilde{a b})^{l+1}\left(\tilde{x}_{0}\right)$ for every $l \in \mathbb{Z}$. 
Proof. (1) Since $\widetilde{\operatorname{rot}}(\tilde{a} \tilde{b})=\frac{6}{5}>1$, we have

$$
\tilde{a}^{2}(\tilde{x})=\tilde{x}+1<\tilde{a} \tilde{b}(\tilde{x})
$$

for every $\tilde{x} \in \mathbb{R}$. This implies the desired inequality.

(2) It follows from (1) that for every $\tilde{x} \in \mathbb{R}$ we have

$$
(\tilde{a b})^{2} \tilde{a}(\tilde{x})=(\tilde{a} \tilde{b})^{2} \tilde{a}(\tilde{x})-2<\tilde{a} \tilde{b}^{3} \tilde{a}(\tilde{x})-2=\tilde{a}^{2}(\tilde{x})=\tilde{x}+1
$$

(3) By substituting $\tilde{b}(\widetilde{a b})^{l+2}\left(\tilde{x}_{0}\right)$ for $\tilde{x}$ in inequality (2), it follows that

$$
(\tilde{a b})^{2} \tilde{a} \tilde{b}(\tilde{a b})^{l+2}\left(\tilde{x}_{0}\right)<\tilde{b}(\tilde{a b})^{l+2}\left(\tilde{x}_{0}\right)+1 .
$$

Since we have

$$
(\tilde{a b})^{2} \tilde{a} \tilde{b}(\tilde{a b})^{2}\left((\tilde{a b})^{l}\left(\tilde{x}_{0}\right)\right)=(\tilde{a b})^{5}\left((\widetilde{a b})^{l}\left(\tilde{x}_{0}\right)\right)+1=(\widetilde{a b})^{l}\left(\tilde{x}_{0}\right)+2,
$$

we obtain the first inequality. Since $l \in \mathbb{Z}$ is an arbitrary integer, it follows that

$$
(\tilde{a b})^{l+2}\left(\tilde{x}_{0}\right)<\tilde{b}(\tilde{a b})^{l+4}\left(\tilde{x}_{0}\right)-1 .
$$

This implies the second inequality. Similarly we have

$$
(\tilde{a b})^{l+4}\left(\tilde{x}_{0}\right)<\tilde{b}(\tilde{a b})^{l+6}\left(\tilde{x}_{0}\right)-1=\tilde{b}(\tilde{a b})^{l+1}\left(\tilde{x}_{0}\right) .
$$

This implies the third inequality.

The following lemma follows from Lemma $2.4(3)$ and the equality $\tilde{a}(\tilde{a b})^{l}\left(\tilde{x}_{0}\right)=$ $\tilde{b}(\widetilde{a b})^{l+4}\left(\tilde{x}_{0}\right)-1$.

Lemma 2.5. For every integer $l \in \mathbb{Z}$, we put

$$
\begin{aligned}
& \tilde{I}_{l}=\left((\tilde{a b})^{l}\left(\tilde{x}_{0}\right),\left(\tilde{b}(\tilde{a b})^{l+2}\right)\left(\tilde{x}_{0}\right)-1\right] \text { and } \\
& \tilde{J}_{l}=\left(\left(\tilde{b}(\widetilde{a b})^{l+2}\right)\left(\tilde{x}_{0}\right)-1,(\tilde{a b})^{l+1}\left(\tilde{x}_{0}\right)\right] .
\end{aligned}
$$

Then we have the following.

1. $\tilde{b}^{-1}\left((\tilde{a b})^{l}\left(\tilde{x}_{0}\right)\right) \in \operatorname{Int}\left(\tilde{J}_{l-4}\right)$ and

1. $(\tilde{b} \tilde{a})\left((\widetilde{a b})^{l}\left(\tilde{x}_{0}\right)\right) \in \operatorname{Int}\left(\tilde{J}_{l+5}\right)$.

2. $\begin{gathered}\tilde{a}\left(\tilde{J}_{l}\right)=\tilde{I}_{l+3} \\ \tilde{L}\left(\tilde{I}_{l}\right)\end{gathered}$

2. $\begin{array}{lllll}\tilde{a}\left(\tilde{J}_{l}\right) & = & \tilde{I}_{l+3}, \\ \tilde{b}\left(\tilde{I}_{l}\right) & \subset & \tilde{J}_{l+3} \text { and } \tilde{b}^{-1}\left(\tilde{I}_{l}\right) \subset \tilde{J}_{l-4} .\end{array}$

We denote by $\left.\phi\left(\widetilde{\pi_{1}^{\text {orb }}\left(\mathcal{O}_{2,3}\right.}\right)\right)$ the subgroup of $\widetilde{\text { omeo }_{+}}\left(\mathbb{S}^{1}\right)$ consisting of lifts of elements of $\phi\left(\pi_{1}^{\text {orb }}\left(\mathcal{O}_{2,3}\right)\right)$ to $\mathbb{R}$. We define a map $\tilde{\theta}$ of $\left.\phi\left(\widetilde{\pi_{1}^{\text {orb }}\left(\mathcal{O}_{2,3}\right.}\right)\right)\left(\tilde{x}_{0}\right)$ onto itself by

$$
\tilde{\theta}\left(\widetilde{\phi(\gamma)}\left(\tilde{x}_{0}\right)\right)=\widetilde{\phi(\gamma)}\left(\widetilde{a b}\left(\tilde{x}_{0}\right)\right)
$$

where $\gamma \in \pi_{1}^{\text {orb }}\left(\mathcal{O}_{2,3}\right)$ and $\widetilde{\phi(\gamma)}$ is a lift of $\phi(\gamma)$ to $\mathbb{R}$. 
Lemma 2.6. The map $\tilde{\theta}$ is well-defined and strictly increasing.

Proof. First we prove that $\tilde{\theta}$ is well-defined. It suffices to show that for $\widetilde{\phi(\gamma)} \in$ $\phi\left(\widetilde{\pi_{1}^{\text {orb }}\left(\mathcal{O}_{2,3}\right)}\right)$ with $\widetilde{\phi(\gamma)}\left(\tilde{x}_{0}\right)=\tilde{x}_{0}$, we have $\widetilde{\phi(\gamma)}\left(\widetilde{a b}\left(\tilde{x}_{0}\right)\right)=\widetilde{a b}\left(\tilde{x}_{0}\right)$.

If $\gamma=\beta^{e_{0}} \alpha \beta^{e_{1}} \ldots \alpha \beta^{e_{n}}$, where $e_{0} \in\{0, \pm 1\}$ and $e_{i} \in\{ \pm 1\}$ for $i \in\{1, \ldots, n\}$, then we have $e_{i} \neq-1$ for $i \in\{0,1, \ldots, n\}$. Indeed if $\left(e_{i}, e_{i+1}, \ldots, e_{n}\right)=$ $(-1,1, \ldots, 1)$ for some $i \in\{0,1, \ldots, n\}$, then it would follow from Lemma 2.5 (1) that

$$
\begin{aligned}
\tilde{b}^{e_{i}} \cdots \tilde{a} \tilde{b}^{e_{n}}\left(\tilde{x}_{0}\right) & \left.=\tilde{b}^{-1}(\tilde{a} \tilde{b})^{n-i}\left(\tilde{x}_{0}\right)\right) \\
& =\tilde{b}^{-1}\left((\tilde{a b})^{n-i}\left(\tilde{x}_{0}\right)\right)+(n-i) \in \operatorname{Int}\left(\tilde{J}_{6(n-i)-4}\right)
\end{aligned}
$$

and hence

$$
\widetilde{\phi(\gamma)}\left(\tilde{x}_{0}\right) \in \operatorname{Int}\left(\tilde{I}_{l}\right) \cup \operatorname{Int}\left(\tilde{J}_{l}\right)
$$

for some $l \in \mathbb{Z}$ by Lemma 2.5 (2), which contradicts the assumption.

Therefore we have $\gamma=\beta^{e_{0}}(\alpha \beta)^{n}$, where $e_{0} \in\{0,1\}$ and it follows from Lemma 2.4 (3) we have $e_{0} \neq 1$. Hence there exists an integer $m \in \mathbb{Z}$ such that

$$
\widetilde{\phi(\gamma)}(\tilde{x})=(\tilde{a b})^{n}(\tilde{x})+m
$$

for every $\tilde{x} \in \mathbb{R}$. We have $n=-5 m$ by the assumption and hence

$$
\widetilde{\phi(\gamma)}\left(\widetilde{a b}\left(\tilde{x}_{0}\right)\right)=(\widetilde{a b})^{-5 m+1}\left(\tilde{x}_{0}\right)+m=\widetilde{a b}\left(\tilde{x}_{0}\right) .
$$

If $\gamma=\beta^{e_{0}} \alpha \beta^{e_{1}} \cdots \alpha \beta^{e_{n}} \alpha$, where $e_{0} \in\{0, \pm 1\}$ and $e_{i} \in\{ \pm 1\}$ for $i \in$ $\{1, \ldots, n\}$, then we have $e_{i} \neq 1$ for $i \in\{0,1, \ldots, n\}$. Indeed if $\left(e_{i}, e_{i+1}, \ldots, e_{n}\right)=$ $(1,-1, \ldots,-1)$ for some $i \in\{0,1, \ldots, n\}$, then it would follow from Lemma 2.5 (1) that

$$
\begin{aligned}
\tilde{b}^{e_{i}} \cdots \tilde{a} \tilde{b}^{e_{n}}\left(\tilde{x}_{0}\right) & \left.=(\tilde{b} \tilde{a})\left(\tilde{b}^{-1} \tilde{a}\right)^{n-i}\left(\tilde{x}_{0}\right)\right) \\
& =(\tilde{b} \tilde{a})\left((\tilde{a b})^{-(n-i)}\left(\tilde{x}_{0}\right)\right) \in \operatorname{Int}\left(\tilde{J}_{-(n-i)+5}\right)
\end{aligned}
$$

and hence

$$
\widetilde{\phi(\gamma)}\left(\tilde{x}_{0}\right) \in \operatorname{Int}\left(\tilde{I}_{l}\right) \cup \operatorname{Int}\left(\tilde{J}_{l}\right)
$$

for some $l \in \mathbb{Z}$ by Lemma 2.5 (2), which contradicts the assumption.

Therefore we have $\gamma=\beta^{e_{0}} \alpha(\beta \alpha)^{n-1}$, where $e_{0} \in\{0,-1\}$ and it follows from Lemma 2.4 (3) that we have $e_{0} \neq 0$. Hence there exists an integer $m \in \mathbb{Z}$ such that

$$
\widetilde{\phi(\gamma)}(\tilde{x})=(\widetilde{a b})^{-(n+1)}(\tilde{x})+m
$$

for every $\tilde{x} \in \mathbb{R}$. We have $n=5 m-1$ by the assumption and hence

$$
\widetilde{\phi(\gamma)}\left(\widetilde{a b}\left(\tilde{x}_{0}\right)\right)=(\widetilde{a b})^{-5 m+1}\left(\tilde{x}_{0}\right)+m=\widetilde{a b}\left(\tilde{x}_{0}\right) .
$$


Next we prove that $\tilde{\theta}$ is strictly increasing. It suffices to show that for $\widetilde{\phi(\gamma)} \in \phi\left(\widetilde{\pi_{1}^{\text {orb }}\left(\mathcal{O}_{2,3}\right)}\right)$ with $\tilde{x}_{0}<\widetilde{\phi(\gamma)}\left(\tilde{x}_{0}\right)$, we have $\tilde{\theta}\left(\tilde{x}_{0}\right)<\tilde{\theta}\left(\widetilde{\phi(\gamma)}\left(\tilde{x}_{0}\right)\right)$.

If $\gamma=\beta^{e_{0}} \alpha \beta^{e_{1}} \cdots \alpha \beta^{e_{n}}$, where $e_{0} \in\{0, \pm 1\}$ and $e_{i} \in\{ \pm 1\}$ for $i \in\{1, \ldots, n\}$, then it follows from Lemma 2.5 (2) that

$$
\widetilde{\phi(\gamma)}\left(\tilde{I}_{0}\right) \subset \tilde{I}_{l} \cup \tilde{J}_{l}
$$

for some non-negative integer $l \in \mathbb{Z}$. This implies that

$$
\widetilde{\phi(\gamma)}\left(\tilde{I}_{1}\right) \subset \tilde{I}_{l+1} \cup \tilde{J}_{l+1}
$$

and hence $\tilde{\theta}\left(\tilde{x}_{0}\right)<\tilde{\theta}\left(\widetilde{\phi(\gamma)}\left(\tilde{x}_{0}\right)\right)$.

If $\gamma=\beta^{e_{0}} \alpha \beta^{e_{1}} \cdots \alpha \beta^{e_{n}} \alpha$, where $e_{0} \in\{0, \pm 1\}$ and $e_{i} \in\{ \pm 1\}$ for $i \in$ $\{1, \ldots, n\}$, then it follows from Lemma 2.5 (2) that

$$
\widetilde{\phi(\gamma)}\left(\tilde{J}_{-1}\right) \subset \tilde{I}_{l} \cup \tilde{J}_{l}
$$

for some non-negative integer $l \in \mathbb{Z}$. This implies that

$$
\widetilde{\phi(\gamma)}\left(\tilde{J}_{0}\right) \subset \tilde{I}_{l+1} \cup \tilde{J}_{l+1}
$$

and hence $\tilde{\theta}\left(\tilde{x}_{0}\right)<\tilde{\theta}\left(\widetilde{\phi(\gamma)}\left(\tilde{x}_{0}\right)\right)$.

The map $\tilde{\theta}$ is $\phi\left(\widetilde{\pi_{1}^{\text {orb }}\left(\mathcal{O}_{2,3}\right)}\right)$-equivariant and we have $\tilde{\theta}^{5}\left(\widetilde{\phi(\gamma)}\left(\tilde{x}_{0}\right)\right)=\widetilde{\phi(\gamma)}\left(\tilde{x}_{0}\right)+$ 1 for every element $\widetilde{\phi(\gamma)}$ of $\left.\phi\left(\widetilde{\pi_{1}^{\text {orb }}\left(\mathcal{O}_{2,3}\right.}\right)\right)$. Since $\phi$ is minimal, $\phi\left(\pi_{1}^{\text {orb }}\left(\mathcal{O}_{2,3}\right)\right)\left(\tilde{x}_{0}\right)$ is dense in $\mathbb{R}$ and hence $\tilde{\theta}$ can be extended to an element of $\widetilde{\text { Homeo }_{+}}\left(\mathbb{S}^{1}\right)$, which we also denote by $\tilde{\theta}$. The homeomorphism $\tilde{\theta}$ is $\phi\left(\widetilde{\pi_{1}^{\text {orb }}\left(\mathcal{O}_{2,3}\right)}\right)$-equivariant and we have $\tilde{\theta}^{5}(\tilde{x})=\tilde{x}+1$ for every $\tilde{x} \in \mathbb{R}$. This gives the desired homeomorphism $\theta \in \mathrm{Homeo}_{+}\left(\mathbb{S}^{1}\right)$.

Acknowledgements The author would like to thank the referee for his/her careful reading of the manuscript and helpful comments.

\section{References}

[1] M. Bucher, An introduction to bounded cohomology, preprint, available at http://www. unige.ch/math/folks/bucher/pdf/KTHnotesIV.pdf.

[2] M. Burger, A. Iozzi and A. Wienhard, Higher Teichmüller spaces: From $\mathrm{SL}(2, \mathbb{R})$ to other Lie groups, in Handbook of Teichmüller theory. Volume IV, 539-618. IRMA Lect. Math. Theor. Phys. 19, Eur. Math. Soc., Zürich, 2014.

[3] D. Calegari, Foliations and the geometry of 3-manifolds, Oxford Mathematical Monographs, Oxford University Press, Oxford, 2007. 
[4] D. Calegari, Ziggurats and rotation numbers, Talk at Low-dimensional Geometry and Topology, Tokyo, September 2012, Slide is available at http://www.is.titech.ac.jp/hyperbolic/index.html.

[5] D. Calegari and A. Walker, Ziggurats and rotation numbers, J. Mod. Dyn. 5 (2011), no. 4, 711-746.

[6] S. Choi, Geometric structures on 2-orbifolds: exploration of discrete symmetry, MSJ Memoirs 27, Mathematical Society of Japan, Tokyo, 2012.

[7] R. Fricke and F. Klein, Vorlesungen der Automorphen Funktionen, Teubner, Leipzig, 1897.

[8] E. Ghys, Groupes d'homéomorphismes du cercle et cohomologie bornée, in The Lefschetz centennial conference, Part III (Mexico City, 1984), 81-106. Contemp. Math. 58, III, Amer. Math. Soc., Providence, RI, 1987.

[9] E. Ghys, Groups acting on the circle, L'Enseignement Mathématique 47, (2001), 329-407.

[10] R. Horowitz, Character of free groups represented in the two dimensional special linear group, Comm. Pure. Appl. Math. 25 (1972), 635-649.

[11] T. Jørgensen, A note on subgroups of $\mathrm{SL}(2, \mathbb{C})$, Quart. J. Math. Oxford Ser. II 28, (1977), 209-212.

[12] K. Mann, Space of surface group representations, Invent. Math., published online: 22 November 2014.

[13] Y. Matsuda, Groups of real analytic diffeomorphisms of the circle with a finite image under the rotation number function, Ann. Inst. Fourier (Grenoble) 59 (2009), no. 5, 1819-1845.

[14] S. Matsumoto, Numerical invariants for semi-conjugacy of homeomorphisms of the circle, Proc. Amer. Math. Soc. 98 (1986), no. 1, 163-168. 